\title{
Cervical acid phosphatase: A biomarker of cervical dysplasia and a potential surrogate endpoint for colposcopy ${ }^{1}$
}

\author{
Olivera Markovic and Nenad Markovic* \\ BioSciCon, Inc., Rockville, MD, USA
}

\begin{abstract}
Background: In 2000, cervical acid phosphatase (CAP) has been recently described as a biomarker labeling abnormal squamous cells on Pap smears (USPTO \#6,143,512). The enzyme activity is presented as a red, granular deposit on a modified Papanicolaou background. This unique property was utilized for development of MarkPap ${ }^{\circledR}$ technology intended for cervical cancer screening.

Material/patients \& methods: We conduct a multicenter, random assignment, assessor blinded, 2-group (test and control), splitsample designed clinical trial on 1,500 subject/specimens to assess safety and efficacy of the new test, in comparison with the control, for cervical cancer screening in standard Pap test environment. Safety is measured with frequency, severity and relation of adverse events. Efficacy is measured with primary endpoints (portion of positive/abnormal specimens detected, and the false negative rate). At the end of the follow-up period (two years) when the study will be completed, other efficacy endpoints such as accuracy (sensitivity/specificity) and predictive values will be added to the method evaluation. Here we present in interim analysis.

Results: In April 2003, the recruitment was completed and the first twelve hundred cases have been evaluated. There was no serious or related adverse event in both groups. Minor, unrelated adverse events were rare and insignificantly distributed in both groups.

Primary endpoints: A: Portion of positive/abnormal specimens detected: Pe (new test) $=0.166$, Ps (Pap control): 0.082; Ps' (ACS reported value for US in year 2000): 0.07. Pe $\geqslant \mathrm{Ps}+\delta$, for $\delta=0.5 \mathrm{Ps}$. B. False negative rate: $\mathrm{Pe}=0.05, \mathrm{Ps}{ }^{\prime}=0.10$. Confidence intervals: 95\% CI: Test [0.148-0.193], Pap control [0.068-0.098]. OR $=2.26 . \chi^{2}=40.69101$ is greater than the critical value of $3.841(P<0.01)$.

Conclusion: We concluded that CAP had added to visibility of Pap test and has enabled cytoscreeners to significantly improve the detection of positive/abnormal specimens and reduce false negative rate. We discuss this unique property of CAP with emphasis on using it as a surrogate endpoint for colposcopy and eventual removal of a cervical lesion that, if untreated, could progress into cancer.
\end{abstract}

Keywords: Biomarker, acid phosphatase, Pap test, cervical cancer, cancer prevention, in vitro diagnostics, medical device, surrogate endpoint

\section{Contributors (clinical or laboratory site and site investigator)}

1. Diagnostic Pathology Services, Inc., Clarksburg,

\footnotetext{
${ }^{1}$ This study was supported in part with the NCI-NIH, SBIR grants R43CA086767, R44CA086767 and R43CA094628.

*Corresponding author: Nenad Markovic, M.D., Ph.D., BioSciCon, Inc., 14905 Forest Landing Circle, Rockville, MD 20850, USA. Tel.: +1 301610 9130; Fax: +1 301610 7662; E-mail: nsmarkovic@comcast.net.
}

MD (laboratory site): James Sundeen, M.D.

2. Department of Pathology, Suburban Hospital, Bethesda, MF (laboratory site): William Smith, Jr., M.D.

3. University of Maryland Health Center, College Park, MD (clinical site): Shelley Parr, M.D.

4. Contemporary Women's Health Care Associates, Bethesda, MD (clinical site): Lewis Townsend, M.D.

5. Women's Health Care Center, Bethesda, MD 
(clinical site): Allan Ross, M.D.

6. Washington Metro Area Ob/Gy Office, Laurel, Washington (clinical site): Ruffus Rosser, M.D.

7. Ob/Gy Office Jed Gould, Laurel, MD (clinical site): Jed Gould, M.D.

8. Montgomery County Language Minority Health Program, Inc., "Proyecto Salud" (clinical site): Michael Morris, M.D.

\section{Introduction}

Pap test was introduced by George Papanicolaou in 1948. The American Cancer Society (ACS) introduced and promoted the Pap test as a screening method for detecting women at risk for cervical cancer. Application of this test in the US resulted in a dramatic reduction of mortality from 11 to $3(>70 \%)$. Similar effects were seen worldwide in countries where this test had been made available. More than 50-M Pap tests are performed annually in the US, with $3.5-\mathrm{M}(7 \%)$ being classified as positive/abnormal. However, there are still 4,500 deaths and 13,000 new cervical cancer cases each year [1]. Studies have shown that $20 \%$ of new cancer cases in the US have never had or had a negative Pap test within 3-5 years before disease progress [2,24]. The high false negative rate $(>10 \%)$ is the major obstacle of Pap test, otherwise the most successful cancer screening test available [1]. This problem persists in spite of recent improvements of Pap test achieved with introduction of liquid-based Pap (LBP) technology [3], automation, HPV testing [4], and better interpretation of results [5]. According to the US Census 2000, among more than 140-M women living in this country, about 100-M are eligible for Pap test. Failures (if permitting cancer to occur in the presence of Pap test can be considered a failure) have occurred among 50-M women who did not chose to have the Pap test checkup, and among the 43-M women who had negative/normal Pap test results. How to find 13,000 among 43-M Pap negative women? How to avoid missing 1,300 abnormal specimens only to find them later as false negatives? Obviously, a more sensitive screening test is needed or a more thorough rescreening of all negative/normal primary screening should be implemented [22]. A highly sensitive test generates many false positives. Therefore, to avoid false alarms and consequences, another, more specific test must be included in the same screening procedure. Why not the Pap test itself?

Between 1960 and 1980, few articles in medical literature described the presence of intracellular acid phos- phatase activity in cervical cancer [6,7], and in vaginal secretions originating from 44 patients suffering from cervical and uterine cancer [8]. This information has never reached major reference cytology books, probably because normal female genital epithelium does not contain acid phosphatase, while detecting this enzyme in vaginal fluids has been used in forensic medicine as an indicator of seminal acid phosphatase [9]. There was no other data in the literature, and cervical acid phosphatase was unnoticed by the scientific community, until 1997 when Markovics raised the question whether this enzyme could play a more important role for detecting cervical dysplasia on Pap smears, and could assist for reducing false-negative readings [10].

Since the description of the new Cervical Acid Phosphatase-Papanicolaou Test (CAP-PAP Test, trademark MARK-PAP ${ }^{\mathrm{TM}}$ ) for visualization of cervical acid phosphatase (CAP) inside abnormal cervical cells on Pap smears [9], it has become possible to explore the nature of this enzyme as a biomarker for cervical dysplasia, and as a possible surrogate endpoint for detection of cervical intraepithelial neoplasia (CIN).

This article is to discuss evidence, collected at an interim analysis (April 2003) of an ongoing clinical laboratory trial. Data is in favour of CAP playing more important role in cervical pathology than it was previously anticipated $[11,12]$.

\section{Cervical Acid Phosphatase as a biomarker}

The MARK-PAP ${ }^{\circledR}$ Test (MPT from now on) is a single-slide, double-staining procedure for demonstration of CAP on the background of a modified Papanicolaou staining. Details of the method are described elsewhere [13]. Overall, MPT is an in vitro diagnostic assay based on chemical reaction (catalysis of phosphate hydrolysis from an aromatic moiety and a simultaneous coupling of this aromatic ring with a diazonium salt) for visualization of the enzyme, and on a modified Papanicolaou staining for visualization of cell morphology. The enzyme activity is presented as a brilliant red intracellular deposit at sites of enzyme activity (Fig. 1: MARK-PAP ${ }^{\circledR}$ Test Images).

The fact that CAP is not present in normal cervical epithelium, as already described by others [6-8], makes the appearance of this pigment in squamous cells a biomarker of cervical cells abnormality. On MPT slides, acid phosphatase activity is also present in monocytes (smears) and endocervical cells (mono- 


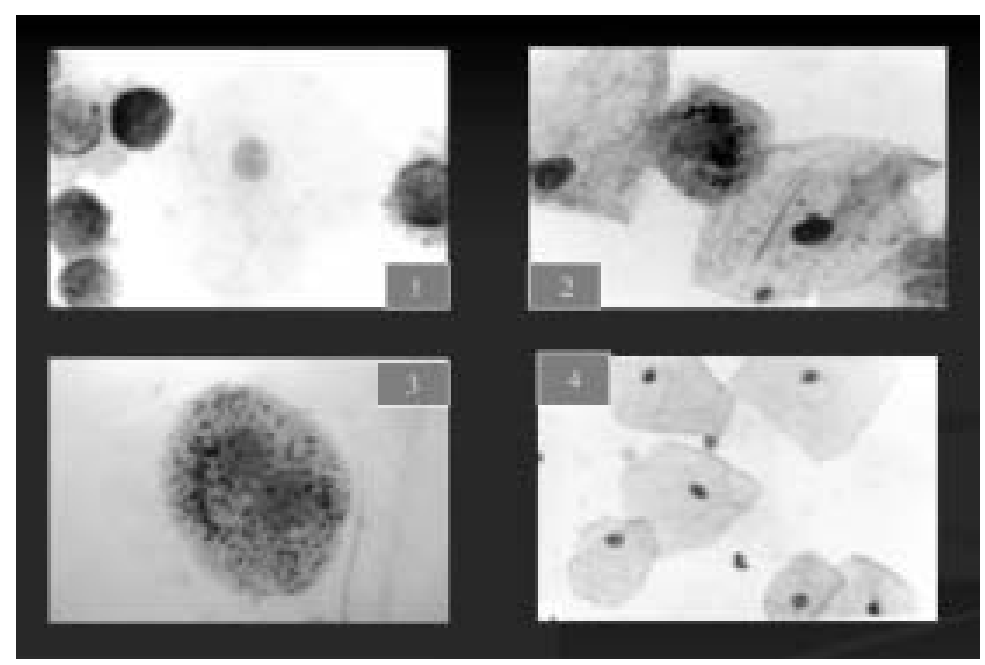

Fig. 1. Mark-Pap test images 1. COMBO control slide. HeLa cells contain CAP (red granular deposit inside cytoplasm). 2. CAP positive abnormal cervical squamous cells surrounded with two CAP negative normally looking cervical squamous cells. 3 . HPV infected, CAP positive, abnormal cervical squamous cell. 4. CAP negative, normal cervical squamous cells.

layers) that serve as internal controls for adequacy of staining and sampling.

Upon our experience from evaluation of 1,500 specimens, CAP is always absent in normal-looking squamous cells, and present in abnormal squamous cells (Fig. 1; 2-3). Since abnormal squamous cells are cytological evidence of cervical dysplasia, finding CAP positive squamous cells on Pap smears or LBP monolayers should have the same effect. At this moment, we consider CAP alone as a biomarker for guiding cytoscreeners' attention, while the interpretation depends upon cytological characteristics of cells stained (in the same procedure) with a modified Papanicolaou technique. For interpretation of findings we recommend the 2001 Bethesda System (Fig. 1; 2-4).

A method that relies on cell characteristics needs reliable standards for quality control and quality assurance. Searching for an external standard, we have found abundant CAP activity in HeLa cell line cells (Kemp Biotechnologies, Frederick, Maryland) [12,15]. We combined this cell line with freshly prepared buccal cells to produce an external standard for CAP activity (HeLa cells) and for cytological staining (buccal cells). The resulting COMBO control slides (Fig. 1; 1), are included in the BioSciCon's MarkPap ${ }^{\circledR}$ Research Kit (Ricca Chemical Company, Arlington, TX). Using these external and internal standards we became convinced that our results are reliable and that CAP indeed is a selective biomarker for abnormal cervical cells [12].

\section{CAP biomarker for improvement of cervical cancer screening}

CAP-PAP test (now MPT) was designed to combine the sensitivity of a chemical marker of cell pathology, with the specificity of Papanicolaou-based staining of cytology, and to produce a test that will be more sensitive than Pap test alone, but will keep the Pap test specificity. This concept was tested in clinical laboratory trials, where the new test was compared with the standard Pap test used for cervical cancer screening.

The BioSciCon sponsored, and NCI-NIH-SBIR (Phase-1 and Phase-2) funded project CAP-PAP Test for Cervical Cancer Screening is a clinical laboratory trial with objectives to assess safety and efficacy of MPT, our new biomarker-based technology, to assist cytoscreeners to improve their own sensitivity for detecting abnormal cervical cells, thus, to reduce false negative results of the Pap test [12].

\subsection{Study design}

Multicenter (seven clinical and two laboratory sites participating as contract research organizations), assignment per order of arrival at each site, split-sample design, assessors blinded, 2-group study (test versus control) to assess the accuracy (sensitivity/specificity) of the new test in comparison with the control (Pap smear or ThinPrep Pap) to select abnormal/positive from normal/negative specimens obtained from 1,500 healthy women who were referred to doctor's offices 
for regular Pap test checkup, and who agreed to participate in this research signing an IRB approved informed consent. Interim analyses were designed to assess the trend of efficacy in comparison with historical control. Failure to maintain improving sensitivity for at least $30 \%$ (level of clinical significance) at any month since the beginning would have resulted in study closure.

\subsection{Data acquisition}

Cervical specimens were obtained at doctor's offices as Pap smears or placed in cell preservative solution for liquid-based Pap (LBP), and sent to laboratory for processing. Each clinical site had to maintain a Clinical Case Report (CCRF) Form for recording the most relevant information for participants at baseline, and the follow-up (two years). Information from CCRF, including the Pap test results, colposcopy assessment, biopsy and histology results, were later filed into a Clinical dbase (Excel).

At laboratory sites, specimens were split and samples were designated for research and for regular Pap test processing.

The conventional Pap test procedure was used for processing and evaluating control samples obtained as smears, and the ThinPrep Pap test was used for samples obtained in solution (LBP). Because the control samples were part of the regular health care service (Pap test checkup), the laboratory returned those results immediately to the clinical sites. MarkPap ${ }^{\circledR}$ test procedure was used for all research samples. A special Laboratory Case Report Form (LCRF) was established to record results of screening. Assessors of research samples were blinded for results of control samples evaluation and vice versa. Assessors blinding was fully implemented to prevent bias of assessment.

The study protocol includes 2-level evaluation of each research slide.

- At the 1st level, a cytotechnologist examines all slides using MPT criteria: monocyte positive - adequate staining; one squamous cell CAP positivethe slide goes to pathologist for review; all squamous cells negative - slide goes to supervisory cytotechnologist for rescreening. Primary screening selects CAP positive/abnormal from negative/normal.

- At the 2nd level, the supervisory cytotechnologist rescreens all negative/normal slides and verifies primary screening or detects false negative slides and refers them to the pathologist.
- At the same 2nd level, a (cyto)pathologist reviews all positive/abnormal slides and false negatives and, using the Bethesda System, gives the interpretation of the clinical condition. This is the final result of screening. Data from LCRF were filed into a Laboratory dbase (Excel).

\subsection{Data analysis}

In April 2003, the clinical and the laboratory dbase were still separated. We have analyzed them as being independent.

\section{a. Primary efficacy endpoint}

The April 2003, Interim Analysis found 1,288 subjects enrolled in the Intent-to-Test (ITT) population. This number represents $86 \%$ of the planned sample size (1,500 subjects). Each specimen was split in two and 2,396 samples were processed. Control samples were completed immediately. ThinPrep procedure was used on $25 \%$ and Pap smear procedure on $75 \%$ of control specimens. All MPT specimens were sent to cytoscreeners for 2-level evaluation. By April 2003, laboratory dbase contained information on 1,088 MPT slides (P-P, per protocol population), but the clinical dbase contained 1,288 Pap test results (ITT) reported to clinical sites.

The primary efficacy endpoint was the portion of positive/abnormal slides detected by either method. Performance was measured by the final result of screening. The threshold was on ASCUS (TBS classification) or AS-US (2001 BS classification). In MPT group, this portion was $16.6 \%(\mathrm{Pe}=0.166)$. In the control group this portion was $8.2 \%$ ( $\mathrm{Ps}=0.082)$. The Pap test detected portion of positive/abnormal slides was higher than the ACS reported standard for US general population $(7 \%)$, probably because of the addition of the ThinPrep controls that are known to be more sensitive [22].

We tested the null hypothesis of equivalence versus a hypothesis of superiority as an alternative. We defined superiority as a $30 \%$ or more increase of detection, but we targeted $50 \%$. The results confirmed the superiority:

$\mathrm{H}_{0}:[\mathrm{Pe}-\mathrm{Ps}] \leqslant \delta$, for $\delta \geqslant 0.5 \mathrm{Ps}$ (targeted) or 0.3Ps (acceptable).

$\mathrm{H}_{\mathrm{A}}: \mathrm{Pe} \geqslant \mathrm{Ps}+\delta$, for $\delta \geqslant 0.5 \mathrm{Ps}$ (targeted) or $0.3 \mathrm{Ps}$ (acceptable).

Results: Superiority accepted: $0.166 \geqslant(0.082+$ $0.024) \geqslant(0.082+0.041)$.

Equivalence rejected: $[0.166-0.082]=0.084 \geqslant$ 0.0246 or 0.041 . 


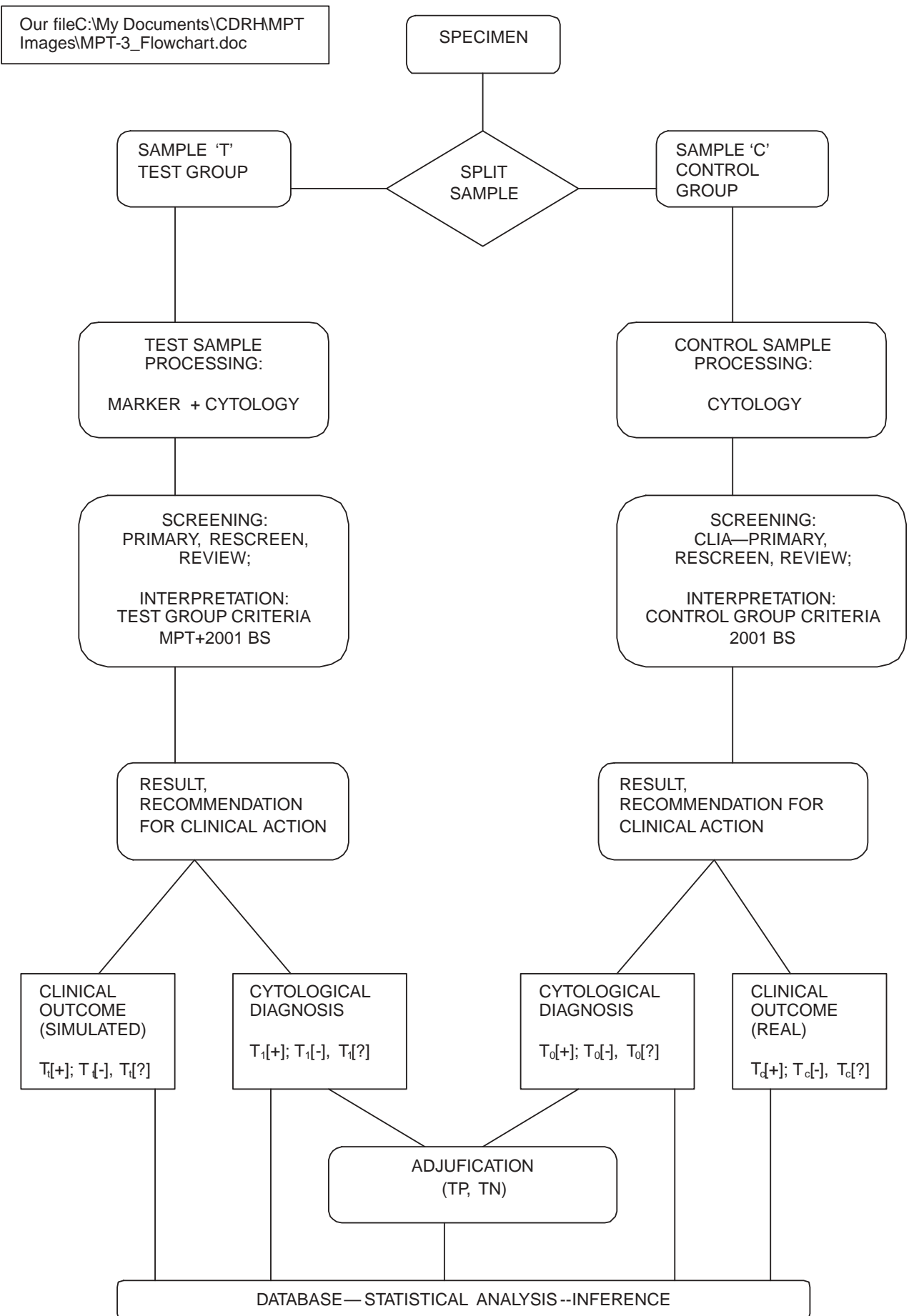

Fig. 2. Split-sample specimen evaluation design for stydy CPS for LBP.

Because historical control (US rates for 2000) was 0.07 , we conclude that MARK-PAP ${ }^{\circledR}$ TEST could indeed increase the number of detected true positive/abnormal specimens.

\section{b. Secondary efficacy endpoints for independent groups}

In this interim analysis we compared the results of reading the test and the control group but as the study 
Table 1

Comparison of screening results between test and control group*

\begin{tabular}{|c|c|c|c|c|c|}
\hline & \multicolumn{3}{|c|}{ Mark-Pap test } & \multicolumn{2}{|c|}{ PAP } \\
\hline & Number & Percent & & Number & Percent \\
\hline $\mathrm{N}$ (subjects) & 1,088 & 100 & & 1,286 & 100 \\
\hline Positive/abnormal & 181 & 16.6 & & 106 & 8.2 \\
\hline Negative/normal & 882 & 81.1 & & 1167 & 90.7 \\
\hline Relative Risk & 0.205 & - & & 0.091 & - \\
\hline $95 \% \mathrm{CI}$ & \multicolumn{3}{|c|}{$0.148-0.193$} & \multicolumn{2}{|c|}{$0.068-0.098$} \\
\hline 95\%CI-difference & \multicolumn{5}{|c|}{$0.060-0.114$} \\
\hline Odds Ratio & \multicolumn{5}{|c|}{2.26} \\
\hline$\chi^{2}$ test & $(\alpha=0.05 ; d f=1)$ & Critical value: & 3,841 & $\Sigma(\mathrm{O}-\mathrm{E})^{2} / \mathrm{E}=40.69$ & $P=0.000000002$ \\
\hline
\end{tabular}

samples came from independent specimens (Table 1). Data were provided from the laboratory and the clinical dbase without coded connectors.

This table indicates that there is a significant difference between cytotechnologist's ability to detect abnormal slides using the new and the control methods for screening. According to this data, the new method is much more sensitive.

\section{c. Decision tree analysis}

We have also used a Decision Tree Model to plot screening data [18]. This model simulates the real-life Pap test screening procedures and provides an opportunity for plotting previous (historic) probabilities. The use of this model permitted regular monitoring of the study progress via interim analyses of endpoints in independent groups (test and control before un-blinding at the end of the study). Results of screening 1,088 MPT specimens were plotted in the following model.

In comparison with the control Pap test (numbers in green boxes), MPT has shown:

A. Increased sensitivity of primary screening as evidenced with:

- Doubling the portion of positive/abnormal slides referred to pathologist (27\%: $12 \%)$

- Reduction in the portion of false negative slides found at rescreening (5\%: 10\%)

- Increase of the portion of disease positive slides (true positive) identified by pathologists (16.6\%: 8.2\%)

- Elimination of false positive primary screening by the pathologist (WNL: 3\%; BCC or benign: $11 \%$ )

Obviously, these effects were related to better visibility of abnormal cells that were labeled with CAP. False alarms were prevented by the second level evaluation using the Bethesda System criteria.
B. Reduced screening time.

Another advantage of using biomarker for quick detection of abnormal cells, was a significant reduction of screening time. Primary screening time was reduced from 6 to 3 min per slide, and rescreen was usually performed for slightly above 1 min per slide [19]. This speed makes 2-level screening, or rapid rescreen of all negative slides an easy and inexpensive task.

\section{d. Safety issues}

We assessed safety as frequency of related adverse events. In vitro diagnostic devices do not have direct contact with humans, therefore, breach of privacy (identification of subjects), un-blinding of assessors, laboratory accidents and similar constitute probable adverse events. None of them was reported until now. Minor bleeding occurred in $2 \%$ and was related to specimen sampling for Pap test. Additional work for preparation of research specimens did not affect subjects. We conclude that the study design was safe and the compliance with protocol has been sufficient to prevent any of expected adverse events to occur.

\section{Surrogate endpoint for cervical dysplasia}

In this report, we have shown some evidence in favor that the new marker of cervical cells abnormality (better sensitivity) combined with the conventional Papanicolaou staining (sustained specificity), should be superior to the conventional staining-alone for early detection of conditions that may evolve into cervical cancer; consequently, for a timely removal of suspect cervical lesions. [1,9] Having shown the selective labelling of abnormal squamous cells with acid phosphatase, we have confirmed the early works of Gross and Kinzie, Malvi and Sirsat, Panazzolo et al. [6-8]. 


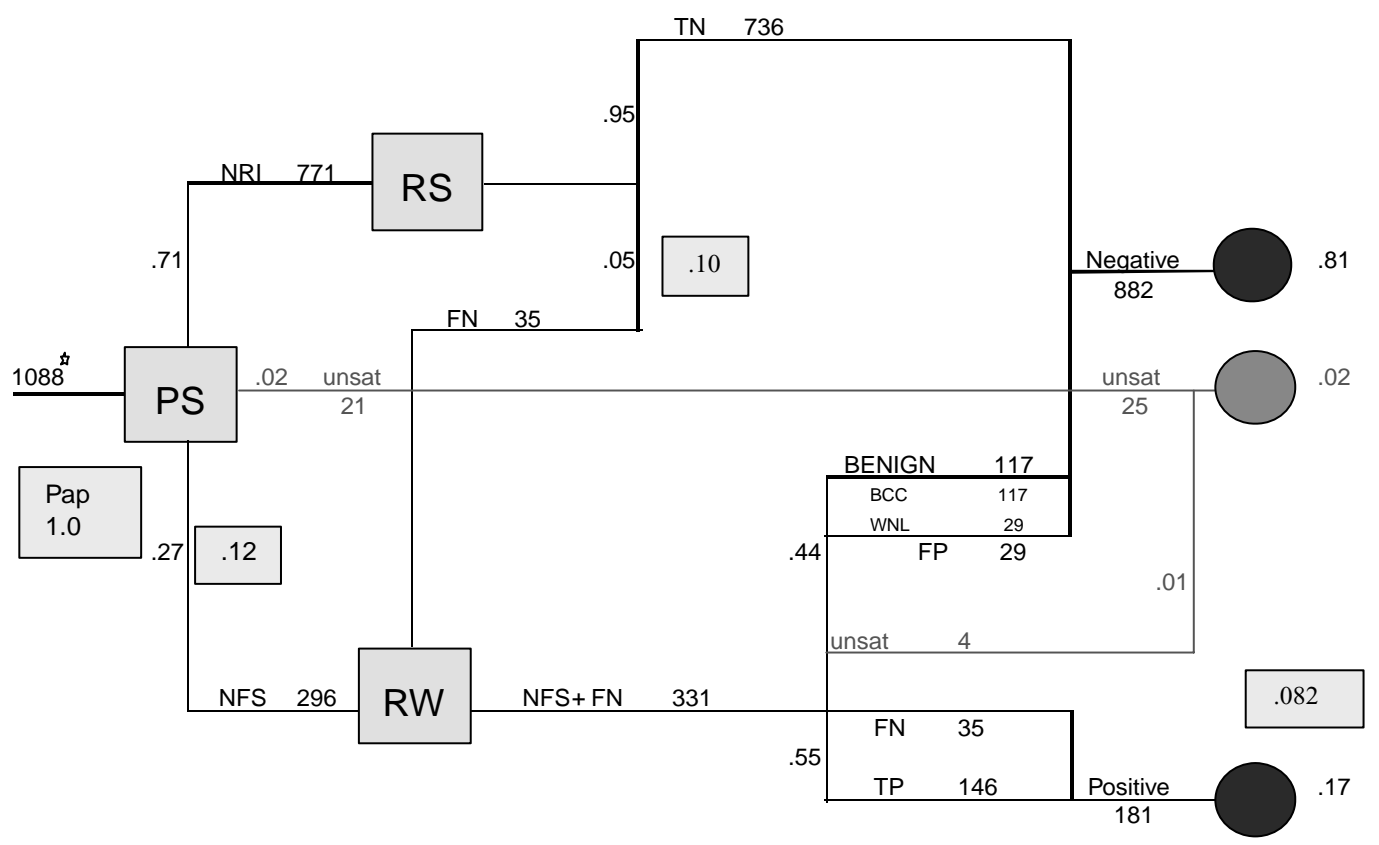

Fig. 3. Laboratory site report - April 2003: MPT Quality Control evaluation and Pap test results. Legend: PS $=$ primary screening (cytotechnologist). RS = rescreening (supervisory cytotechnologist). RW = review (pathologist). Green boxes = prior probabilities (established in the same laboratory for the same population but using the Pap test only). NFS = need further screening (positive/abnormal sent to the reviewer for cytological evaluation). NRI = no review indicated (negative sent to rescreener for verification). FN = false negative, missed NFS found at $100 \%$ rescreen. $\mathrm{FP}=$ false positive detected as WNL at review. $\mathrm{TP}=$ true positive, determined as ASCUS and above by the reviewer. $\mathrm{TN}=$ true negative, determined as benign by the reviewer and NRI verified by the rescreener.

With regard to screening for cervical cancer and the Pap test obstacles with the false negative rates, we would like to point that our study shows a false negative rate of $4-5 \%$ while the reference controls show $10 \%$ for Pap test overall [2], 13\% for ThinPrep [22] or $18 \%$ for conventional Pap smears [23]. Better visibility of abnormal cells on slides is an obvious advantage of using a biomarker such as CAP.

The goal of this report was to indicate to cervical acid phosphatase as to a neglected biomarker with potentials in cervical cancer screening. When the trail will be over, the screening results will be compared with cytological standard verified with colposcopy/biopsy and histology, and with clinical outcomes (disease absence/progression within two years). We expect the final results to confirm this initial favourable data.

In our study CAP was selectively seen in abnormal squamous cells, its presence significantly increased the portion of detected positive/abnormal specimens and reduced the false negative rates. It was achieved when Pap-based Bethesda System terminology was used for evaluation of clinical condition indicated as abnormal with CAP presence. It is too early to discuss whether CAP alone could be of use for cervical cancer screen- ing. However, we believe, it would be soon a rewarding challenge (for us and for others) to explore whether cervical acid phosphatase has the potentials to become a surrogate endpoint for making clinical decisions on who should be referred to further diagnostic procedures intended for elimination of early cervical lesions that could progress into cancer.

To initiate this discussion we are presenting our views on this issue. At this moment, our data show that CAP has at least two of three characteristics required for surrogate endpoints [20].

- On MPT specimens, cervical acid phosphatase detects cells with abnormalities indicative of cervical intra-epithelial neoplasia (CIN). The presence of this lesion is a well-established risk factor for cervical cancer $[1,21]$.

- The MARK-PAP ${ }^{\circledR}$ Test (with accessories) clearly describes procedures for visualization of cervical acid phosphatase inside abnormal cervical cells, provides tools (reagents, controls and instructions) for achieving this goal, and criteria for converting cytological findings into clinical actions $[12,15]$.

At this moment, we do not have enough knowledge of the relation between cervical acid phosphatase and 
the clinical outcomes to recommend this enzyme as a surrogate endpoint for surgery, but we are accumulating evidence that could provide better information on the real value of cervical acid phosphatase as a surrogate endpoint to indicate necessity for further screening (colposcopy and biopsy/histology).

\section{Conclusion \& prospective}

We present evidence accumulated within a predetermined interim period of our clinical trial. This evidence is in support of CAP being an efficient biomarker for enhancing visibility of abnormal squamous cells on Pap smears and monolayers of LBP. This new test that allows simultaneous visualization of the new CAP marker and cell morphology, has been found superior to Pap test-alone for detecting cervical specimens with epithelial cell abnormalities.

Data are indicative that CAP, when more evidence is available, could be considered as a surrogate endpoint for clinical decision to refer a test positive subject to colposcopy and further diagnostic and therapeutic procedures for prevention of cervical cancer.

If and when CAP became considered as a surrogate endpoint, an entirely new approach to cervical cancer screening might be considered: search for methods to detect cervical acid phosphatase in vaginal secretions including, but not limited to immunological, biochemical and molecular methods.

\section{Acknowledgment}

The authors wish to acknowledge continuing support by Dr. Mukesh Verma, Program Director, Cancer Biomarkers Research Group, Cancer Prevention, NCI, NIH. The authors wish to acknowledge data management assistance provided by Dr. Vladan Posarac, and the technical assistance provided by Ms. Milena Simic, both from BioSciCon, Inc., Rockville, MD.

\section{References}

[1] American Cancer Society: New Guideline for Early Detection of Cervical Cancer, CA Caner I Clin 52 (2001), 342-362.

[2] CDC Report: Incidence of Pap Test abnormalities Within 3
Years of a Normal Pap Test - United states, 1991-1998. M<WR, 49 (2000), 1001-1003 (http://www.cdc.gov/mmwr/ review).

[3] Cytyc Corp Home Page (http://www.thinprep.com).

[4] Digene Home Page (http://www.digene.com).

[5] TriPath Imaging Home Page (http://www.tripathimaging. com).

[6] S.G. Malvi and S.M. Sirsat, A cytochemical study of acid phosphatase in carcinoma of the cervix uteri, The Indian Journal of Cancer 11 (1974), 81-87.

[7] S.J. Gross and G. Kinzie, Cytochemistry of benign and malignant squamous epithelium of the cervix uteri, Obst. and Gynecol. 15 (1960), 261-279.

[8] A. Panazzolo, L Bergantino and S. Arrotta et al., Gli enzimi lisosomiali nella patologia neoplastica del collo dell'utero, Min. Gin. 30 (1978), 1123-1145, (Italian).

[9] N. Markovic and O. Markovic, CAP-PAP Test, USPTO No. 6,143,512 (November 2000).

[10] O. Markovic and N. Markovic, May acid phosphatase decrease Pap test negative readings? J Nat Cancer Inst 89 (1997), 1459.

[11] O. Markovic, CAP-PAP Test for Cervical Cancer Screening, SBIR Phase-1 Report, in NIH-NCI-SBIR Phase-2 Grant Application "CAP-PAP Test for Cervical cancer Screening 2," SBIR Phase-2 Grantr, NIH \#1R44CA86767-02.

[12] O. Markovic, CAP-PAP Test for Cervical Cancer Screening 2,SBIR Phase-2 Grant Application, NIH \#1R44CA86767-02.

[13] O. Markovic, N. Markovic and M. Belledonne, Cervical Acid Phosphatase - Papanicolaou (CAP-PAP) Test, J Histotechnology 22 (1999), 43-47.

[14] O. Markovic and N. Markovic, Acid phosphatase in cervical smears (CAP-PAP test), Arch Oncol 6 (1998), 137-139.

[15] N. Markovic and O. Markovic, CAP-PAP Test Kit, USPTO Application No. 10/339,760 (January 2003).

[16] N. Markovic, CAP-PAP Test for Specimens Collected in Solution, NIH-SBIR Grant \# 1R43CA94628-01.

[17] N. Markovic, MARK-PAP Test Kit, SBIR-NIH Grant Application \#1 R43CA1017292-01.

[18] B. Dawson-Saunders and R.G. Trapp, Basic \& Clinical Biostatistics, Lange Medical Book, Norwalk CN, 1994, pp. 249267.

[19] MARK-PAP ${ }^{T M}$ Technology Licensing Package, Executive Summary, BioSciCon, Rockville, MD, 2003.

[20] S. Srivastava and J.A. Wagner, Surrogate endpoints in medicine, Disease Markers 18 (2002), 39-40.

[21] T.C. Wright, T. Cox and S. Massad et al., for the 2001 ASCCP Consensus Conference. Consensus Statement: ASCCP 2001 Consensus Guidelines for the Management of Women with Cervical Cytological Abnormalities, JAMA 287 (2002), 2120 2129.

[22] L.R. Rowe, C.J. Marshall and J.S. Bentz, One hundred percent thorough quality control rescreening of liquid-based monolayers in cervicovaginal cytopathology, Cancer 25:96(6) (2002), 325-329.

[23] M. Arbyn and U. Schenck, Detection of false negative Pap smear by rapid reviewing. A meta-analysis, Acta Cytol. 44(6) (2000), 949-957.

[24] Cervical Cancer. NIH Consensus Statement 1996 Apr 1$3 ; 14(1): 1-38$. 


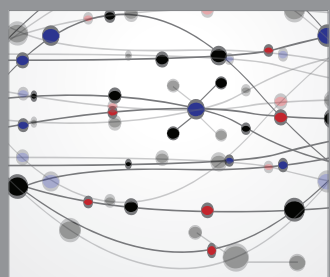

The Scientific World Journal
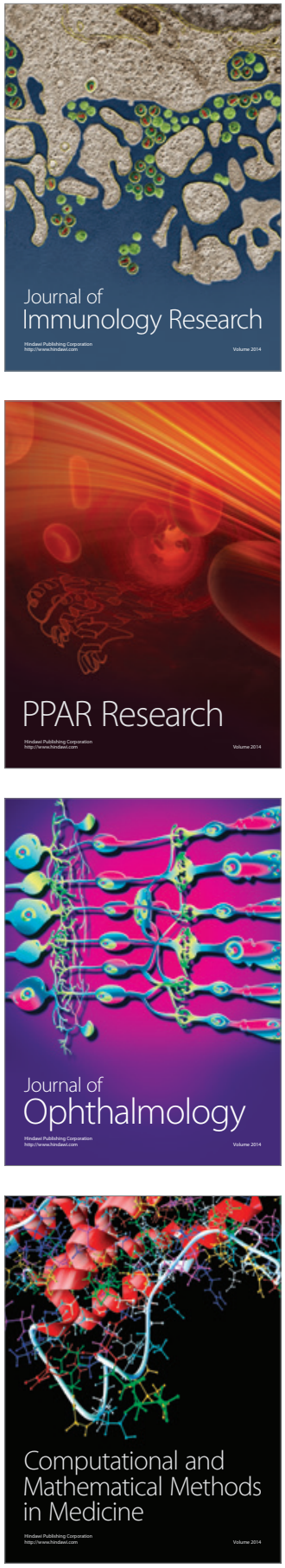

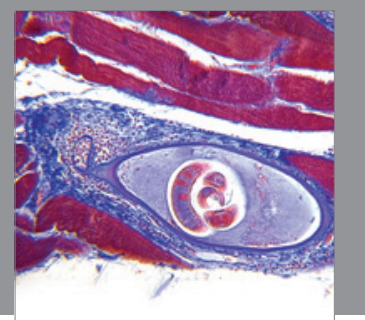

Gastroenterology

Research and Practice
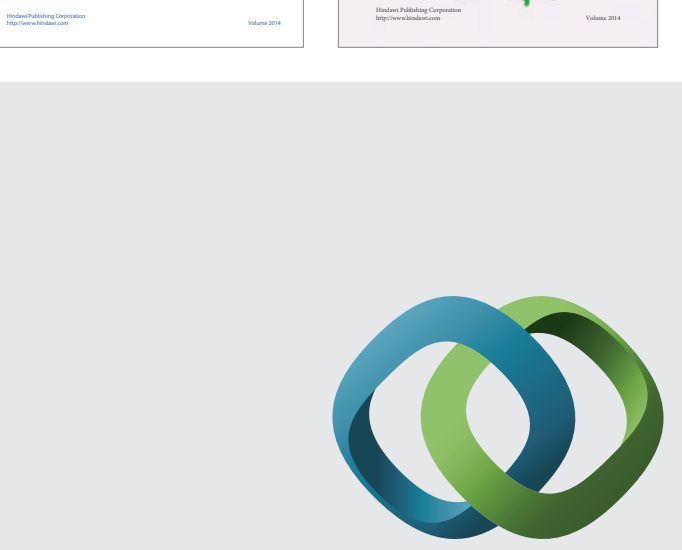

\section{Hindawi}

Submit your manuscripts at

http://www.hindawi.com
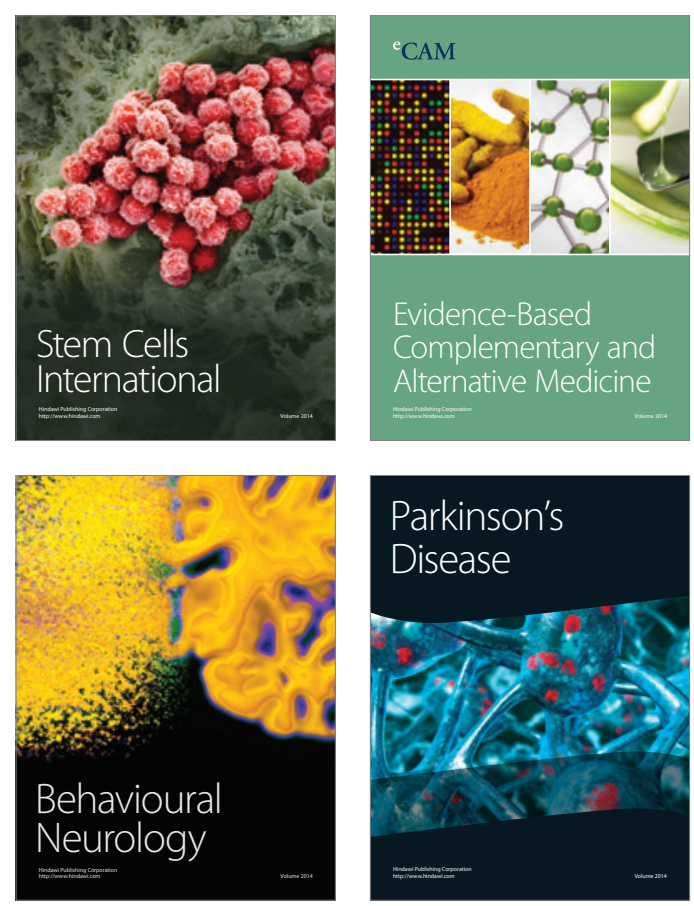

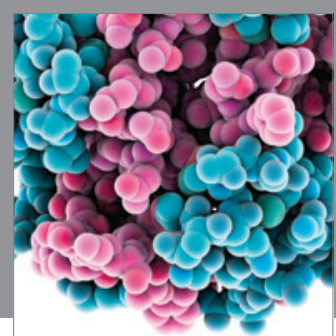

Journal of
Diabetes Research

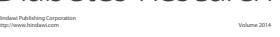

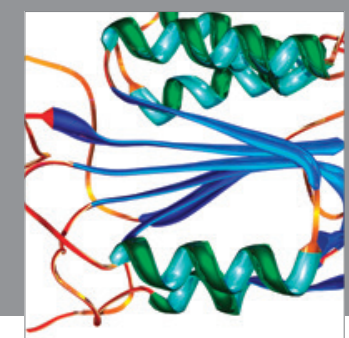

Disease Markers
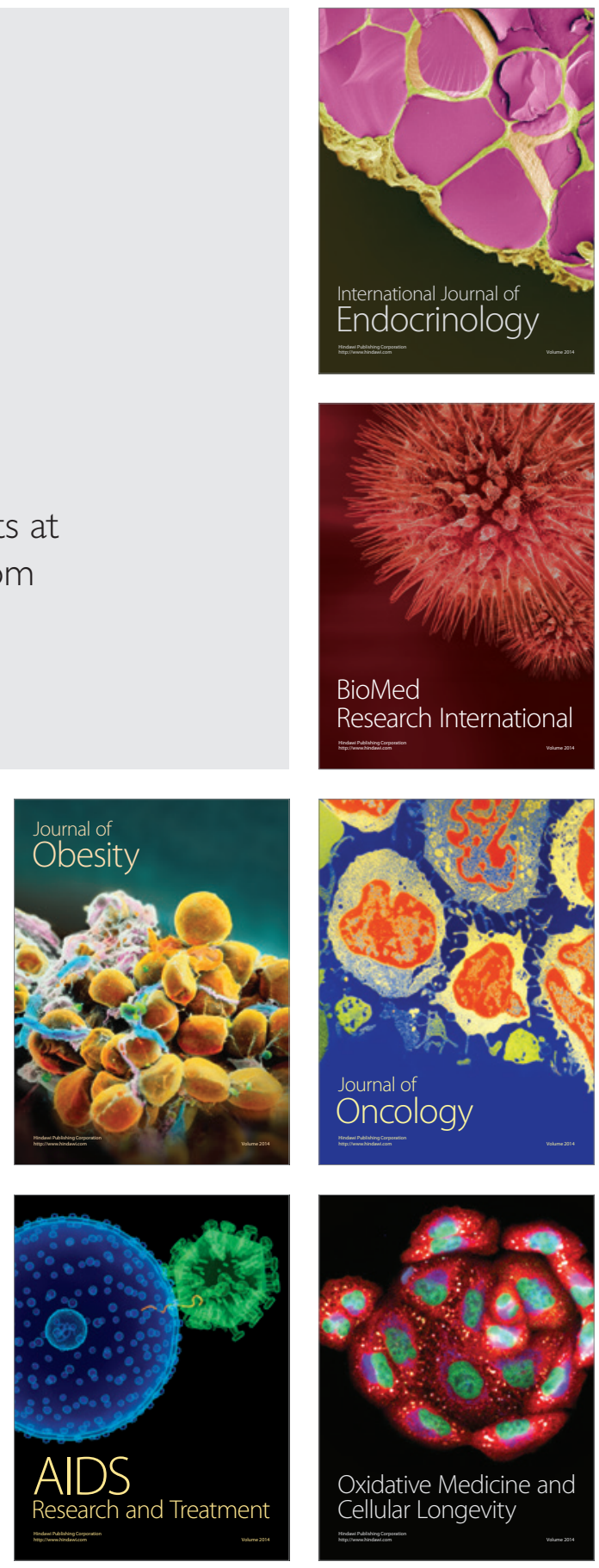